\title{
Equalizadores Turbo de Baixa Complexidade para Canais de $\mathrm{HF}$
}

\author{
Robson F. de Moraes, Juraci F. Galdino e Ernesto L. Pinto.
}

\begin{abstract}
Resumo-Este trabalho investiga a aplicação de equalizacão turbo a canais de HF. Propõe-se dois algoritmos de equalização linear baseados no critério MMSE (Minimum Mean-Square Error), obtidos a partir de modificacões num esquema MMSE anteriormente proposto por outros autores. São apresentados resultados de avaliação de desempenho via simulação computacional, usando como referência o padrão MIL-STD-188-110A/B, que prevê formas de onda para modems seriais em HF. A análise destes resultados mostra que não ocorre degradação significativa de desempenho com as modificações introduzidas. Por outro lado, uma análise comparativa de complexidade computacional mostra que uma das propostas aqui apresentadas produz significativa redução na complexidade do equalizador.
\end{abstract}

Palavras-Chave-Equalização turbo, canais de HF, equalizadores turbo de baixa complexidade, equalizadores turbo MMSE, estimação de canal.

Abstract - This work deals with the application of turbo equalization to HF channels. Two algorithms for linear equalization using the MMSE criterion are here presented, which are obtained from modifications of an MMSE scheme previously proposed by other authors. Several results of performance evaluation by computer simulation are presented, taking the MIL-STD$188-110 \mathrm{~A} / \mathrm{B}$ standard for serial modems as a reference. These results show that the modifications here proposed keep the BER performance of the equalizers almost unaffected. On the other hand, one of these modifications is shown to give rise to a significant reduction of computational complexity.

Keywords-Turbo equalization, HF channels, low-complexity turbo equalizers, MMSE turbo equalizers, channel estimation.

\section{INTRODUÇÃO}

A comunicação rádio na faixa de HF (de High Frequency), caracterizada pelo uso de frequências na faixa de $3-30 \mathrm{MHz}$, oferece um forte atrativo por ser capaz de cobrir grandes distâncias devido aos diferentes modos de propagação presentes. Em particular, o interesse nesta faixa surge devido à propagação por ondas celestes (ionosféricas) que, através do fenômeno de reflexão virtual (múltiplas refrações) permite a comunicação BLOS (de Beyond Line of Sight).

Porém este canal apresenta: propagação multicaminho, desvanecimento e ruídos aditivos. Em cada componente multicaminho ocorre ainda desvanecimento variante no tempo, devido a irregularidades na ionização, produzindo o efeito de espalhamento Doppler.

Uma forma de mitigar os efeitos da IES (Interferência Entre Símbolos) é o uso de equalizadores adaptativos no receptor,

Robson F. de Moraes, Divisão de Engenharia, Fábrica de Material de Comunicações e Eletrônica, Indústria de Material Bélico do Brasil - IMBEL, Rio de Janeiro, Brasil, E-mail: robson_moraes@ hotmail.com. Juraci F. Galdino e Ernesto L. Pinto, Departamento de Engenharia Elétrica, Instituto Militar de Engenharia, Rio de Janeiro, Brasil, E-mails: galdino@ime.eb.br, ernesto@ime.eb.br. os quais procuram combater os efeitos de dispersão do canal, buscando estimativas dos símbolos transmitidos a partir de algum processamento mais elaborado das observações. Nos estudos de formas eficazes de equalização, diversas técnicas de equalização linear e não-linear têm sido propostas ao longo das últimas décadas, destacando-se, pelas suas características de desempenho, os equalizadores não lineares, tais como os do tipo MLSE (de Maximum Likelihood Sequence Estimator) e do tipo DFE (de Decision Feedback Equalizer) [1]. Tem sido mais frequiente o emprego prático de equalizadores DFE, pelo fato de apresentarem menor complexidade computacional que os equalizadores MLSE, com perda de desempenho considerada compensadora diante da redução de complexidade [2].

O receptor ótimo no sentido da taxa de erro, aplicado a um canal variante no tempo como o caso da faixa de HF, deve realizar conjuntamente as tarefas de estimação de canal, equalização e decodificação. Porém, tal tarefa torna-se inviável na prática devido à alta complexidade computacional apresentada pelos algoritmos. Tem sido comum, então, na implementação prática de receptores aplicar os processamentos de equalização e decodificação de forma independente. Nos esquemas mais simples, o equalizador produz decisões abruptas sobre os símbolos recebidos, para que os mesmos sejam mapeados em bits detectados e possam, finalmente, ser processados pelo decodificador.

Este processo de decisão abrupta tomada pelo equalizador, no entanto, destrói informação suave acerca da confiabilidade da observação, produzindo a mesma saída para observações com diferentes níveis de confiabilidade.

Admitindo que os bits transmitidos são equiprováveis e que as amostras recebidas também o são, a informação suave associada a uma determinada amostra pode ser entendida como uma medida da razão de probabilidades a posteriori correspondente, $\frac{P\left(c_{k}=0 \mid o b s\right)}{P\left(c_{k}=1 \mid o b s\right)}$ onde $c_{k}$ é o bit sobre o qual se calcula a razão e obs é o conjunto de amostras recebidas. Assim sendo, esta informação pode ser explorada por um decodificador obtendo-se então um melhor desempenho de taxa de erro (decodificação baseada em decisão suave) [3].

Baseado na invenção dos códigos turbo ocorreu o surgimento do chamado processamento turbo de recepção. Este processamento se caracteriza pela troca de informações entre módulos de um processo que cooperam para a obtenção do resultado final através de uma troca de informação suave semelhante à originalmente explorada na decodificação turbo.

A aplicação do então chamado "princípio turbo"pode ser vista, hoje em dia, nas mais diversas técnicas associadas às comunicações. Pelo especial interesse neste trabalho, destacamos a aplicação deste princípio à equalização, gerando o que passou-se a chamar de turbo equalização. 
A turbo equalização é, assim, um processo de equalização e decodificação conjunta, conduzido de forma iterativa, proporcionando a troca de informação entre os blocos equalizador e decodificador de um sistema receptor.

Entretanto o turbo equalizador ótimo, no sentido da máxima probabilidade a posteriori de símbolo, requer o uso do algoritmo BCJR-MAP ${ }^{1}$ [5] (Maximum A Posteriori), que tem complexidade computacional impeditiva para o uso de modulações multi-níveis em canais com grande espalhamento de retardos, como é o caso nas transmissões com elevada eficiência espectral em canais de HF.

Em função deste impedimento, pesquisas têm sido realizadas no sentido de se obter equalizadores SISO (de SoftInput Soft-Output) com baixa complexidade e pouca perda de desempenho em relação aos equalizadores baseados no algoritmo BCJR. Surgiram os equalizadores SISO baseados em filtros lineares, que foram introduzidos em [6].

De particular interesse, tem-se os algoritmos que fazem uso do critério MMSE (de Minimum Mean Square Error), baseando-se em filtros lineares com coeficientes variantes no tempo. Tais algoritmos apresentam bom desempenho, quando comparados ao esquema ótimo, porém com uma complexidade reduzida.

Neste artigo são propostas duas alternativas de algoritmos baseados no critério MMSE, avaliando-se seu desempenho através de simulação computacional tomando como referência o padrão MIL-STD-188-110A/B.

\section{DefiniÇÃo do Sistema EM Estudo}

O modelo de sistema de comunicação aqui estudado tem seu diagrama em blocos ${ }^{2}$ representado na Figura 1. No receptor

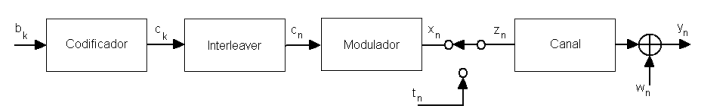

(a)

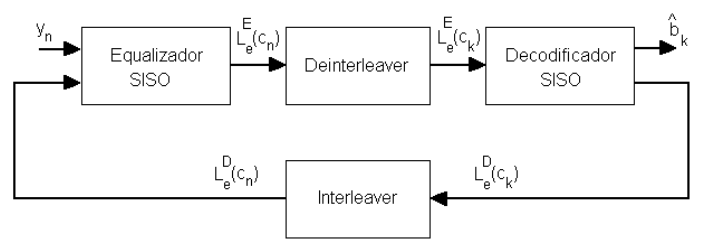

(b)

Fig. 1. Modelo de sistema de comunicação: (a) Transmissor e (b) Receptor Iterativo.

da Figura 1 são utilizados um equalizador e um decodificador do tipo SISO. Tais componentes são assim chamados por trabalharem com informação suave na entrada e na saída.

O equalizador SISO recebe, juntamente com as observações do canal, a informação suave a priori $L_{e}^{D}\left(c_{n}\right)$ sobre os bits codificados provenientes do decodificador na iteração anterior.

\footnotetext{
${ }^{1} \mathrm{~A}$ abreviatura BCJR provém das iniciais dos autores do artigo que propõe este decodificador [4].

${ }^{2}$ Por simplicidade de representação não foram acrescentados ao diagrama os filtros de transmissão e de recepção
}

A partir dessas informações gera a informação suave de saída $L_{e}^{E}\left(c_{n}\right)$. Os símbolos conhecidos transmitidos têm a função de gerar o sincronismo entre transmissor e receptor, e, em casos de canais desconhecidos, auxiliar a estimação de parâmetros do mesmo.

Um requisito do princípio turbo é que a informação suave de saída de um módulo SISO não pode ser função da informação suave a priori para um mesmo bit codificado. Se o equalizador for baseado no critério MAP vale a relação

$$
L_{e}^{E}\left(c_{n}\right)=L^{E}\left(c_{n}\right)-L_{e}^{D}\left(c_{n}\right)
$$

onde $L^{E}\left(c_{n}\right)$ é a LLR (de Log-Likelihood Ratio) a posteriori calculada pelo equalizador, $L_{e}^{D}\left(c_{n}\right)$ é a LLR a priori proveniente do decodificador calculada na iteração anterior, e $L_{e}^{E}\left(c_{n}\right)$ é chamada de informação extrínseca ou LLR extrínseca.

Como visto na Figura 1, um conjunto de $N$ bits $b_{k}$ é passado através de um codificador gerando $N_{\text {int }}=N \cdot R_{c}^{-1}$ bits codificados $c_{k}$, onde $R_{c}$ é denominado a taxa do codificador $\left(0<R_{c}<1\right)$. Essa seqüência de bits codificados alimenta um entrelaçador, que rearranja a ordem temporal do conjunto, fornecendo uma sequiência de bits $c_{n}$, onde para denotar o rearranjo temporal troca-se o índice subscrito. Os bits codificados são então modulados, sendo cada grupo de $Q$ bits mapeado em um símbolo de acordo com uma modulação $2^{Q}$-ária, gerando um conjunto de $\frac{N_{i n t}}{Q}$ símbolos $x_{n}$. Antes de passar pelo canal, a sequiência de símbolos desconhecidos $x_{n}$ é multiplexada com uma seqüência de $N_{t}$ símbolos $t_{n}$, conhecidos pelo receptor, o que gera a sequiência de símbolos $z_{n}$ que é transmitida pelo canal. O sinal recebido é corrompido por ruído aditivo complexo representado por amostras $w_{n}$, gerando os símbolos recebidos $y_{n}$.

Considerando que os filtros de transmissão e de recepção obedecem ao critério de Nyquist, admitindo-se que $\int_{-\infty}^{\infty} f_{R X}^{2}(t) d t=1$ (onde $f_{R X}$ é a resposta ao impulso dos filtros), e que a taxa de amostragem do receptor é igual à taxa de símbolos com sincronismo perfeito, o equivalente do canal em tempo discreto se torna

$$
\mathbf{h}_{n}=\left[h_{n, 0} h_{n, 1} \ldots h_{n, L-1}\right]^{T}
$$

onde L é o comprimento do equivalente do canal medido em intervalos de símbolo.

A sequiência de amostras na entrada do equalizador é dada por:

$$
y_{n}=\sum_{i=0}^{L-1} h_{n, i} x_{n-i}+w_{n}
$$

onde $w_{n}$ são amostras do ruído Gaussiano complexo filtrado, com $\sigma_{w}^{2}=N_{0}$ e $\mathbf{x}_{n}=\left[\begin{array}{ll}x_{n} & x_{n-1} \cdots x_{n-L+1}\end{array}\right]^{T}$ é o vetor de símbolos transmitidos.

Conforme o modelo de Watterson, cada coeficiente $h_{n, i}$ deve possuir um espectro Doppler Gaussiano. A geração destes foi feita através do método de Monte-Carlo [7].

Conforme representado no item (b) da Figura 1, um receptor utilizando processo iterativo tem a necessidade de trabalhar com blocos que recebam e forneçam informação suave (SISO). Como o foco do presente artigo são os equalizadores, foi decidido fixar-se um tipo somente de decodificador SISO. O 
decodificador que apresenta desempenho ótimo no sentido da taxa de erro é o que utiliza o critério MAP.

Foi então escolhido o decodificador BCJR/MAP proposto em [4], apesar de ter complexidade alta por se tratar de um decodificador baseado em treliça. Esta escolha porém garante majorar o desempenho do sistema utilizando-se os equalizadores trabalhados neste texto. Uma implementação do decodificador BCJR é apresentada em [3].

\section{Algoritmo MMSE}

Em [8] foi deduzido um algoritmo MMSE para canais variantes desconhecidos.

Denotando o vetor de coeficientes da resposta ao impulso do canal no instante $n$ por

$$
\mathbf{h}_{n} \triangleq\left[h_{n, 0} h_{n, 1} \cdots h_{n, L-1}\right]^{T}
$$

pode-se definir uma matriz de convolução, porém $\mathbf{h}_{n}$ não é conhecido e deve-se utilizar uma estimativa $\hat{\mathbf{h}}_{n}$. Assim tem-se uma matriz de convolução estimada, dada por:

$$
\hat{\mathbf{H}}_{n} \triangleq\left[\begin{array}{cccccc}
\hat{h}_{n, L-1} & \hat{h}_{n, L-2} & \ldots & \hat{h}_{n, 0} & \cdots & 0 \\
0 & \hat{h}_{n, L-1} & \hat{h}_{n, L-2} & \cdots & \cdots & 0 \\
& & & \ddots & & \\
0 & & \ldots & 0 & \cdots & \hat{h}_{n, 0}
\end{array}\right]
$$

Quando o canal é estimado pode-se definir um erro que engloba uma parcela devido ao ruído e outra devido ao erro de estimação do canal que é definido como

$$
e_{n}=y_{n}-\sum_{i=0}^{L-1} \hat{h}_{n, i} z_{n-i}
$$

cuja variância é aqui denotada por $\sigma_{e, n}^{2}$. Note-se que esta variância depende do índice $n$.

A estimativa de símbolos transmitidos usando o critério MMSE é dada por:

$$
\begin{aligned}
\hat{z}_{n}=E\left(z_{n}\right)+ & \operatorname{Cov}\left(z_{n}, \mathbf{y}_{n}\right) \operatorname{Cov}\left(\mathbf{y}_{n}, \mathbf{y}_{n}\right)^{-1}\left[\mathbf{y}_{n}-E\left(\mathbf{y}_{n}\right)\right] \\
\text { Definindo } \mathbf{e}_{n} & \triangleq\left[e_{n-N_{2}} \cdots e_{n} \cdots e_{n+N_{1}}\right]^{T} \text { tem-se: } \\
\boldsymbol{\Sigma}_{n} & \triangleq \operatorname{Cov}\left(\mathbf{y}_{n}, \mathbf{y}_{n}\right) \\
& =E\left(\mathbf{y}_{n} \cdot \mathbf{y}_{n}^{H}\right)-E\left(\mathbf{y}_{n}\right) E\left(\mathbf{y}_{n}^{H}\right) \\
& =\hat{\mathbf{H}}_{n} \operatorname{Cov}\left(\mathbf{z}_{n}, \mathbf{z}_{n}\right) \hat{\mathbf{H}}_{n}^{H}+\mathbf{\Psi}_{n} \\
& =\hat{\mathbf{H}}_{n} \mathbf{V}_{n} \hat{\mathbf{H}}_{n}^{H}+\mathbf{\Psi}_{n}
\end{aligned}
$$

onde $\boldsymbol{\Psi}_{n} \triangleq \operatorname{diag}\left(\left[\sigma_{e, n-N_{2}} \cdots \sigma_{e, n} \cdots \sigma_{e, n+N_{1}}\right]\right)$ é a matriz covariância do erro $e_{n}$.

Pode-se definir a estimativa do equalizador e o vetor de coeficientes do filtro linear como sendo:

$$
\begin{aligned}
\hat{z}_{n}= & \overline{\mathbf{z}}_{n}+v_{n} \mathbf{s}^{H} \boldsymbol{\Sigma}_{n}^{-1}\left[\mathbf{y}_{n}-\overline{\mathbf{y}}_{n}\right] \\
= & \overline{\mathbf{z}}_{n}+v_{n} \mathbf{f}_{n}^{H}\left[\mathbf{y}_{n}-\overline{\mathbf{y}}_{n}\right] \\
& \mathbf{f}_{n} \triangleq \boldsymbol{\Sigma}_{n}^{-1} \mathbf{s}
\end{aligned}
$$

Usando a restrição de que a informação extrínseca $L_{e}^{E}\left(c_{n, j}\right)$, que é função de $\hat{x}_{n}$ e da informação a priori $L\left(c_{n, j^{\prime}}\right)$ para todo $j^{\prime} \neq j$, não pode ser função de $L\left(c_{n, j}\right)$.

$$
\begin{aligned}
\hat{z}_{n} & =0+1 \cdot \mathbf{f}^{\prime}{ }_{n}^{H}\left[\mathbf{y}_{n}-\overline{\mathbf{y}}_{n}+\left(\bar{z}_{n}-0\right) \mathbf{s}\right] \\
\mathbf{f}^{\prime}{ }_{n} & \left.\triangleq \mathbf{f}_{n}\right|_{v_{n}=1}=\left[\boldsymbol{\Sigma}_{n}+\left(1-v_{n}\right) \mathbf{s s}^{H}\right]^{-1} \mathbf{s}
\end{aligned}
$$

Utilizando o lema de inversão de matrizes [9]

$$
\begin{aligned}
\mathbf{f}_{n}^{\prime} & =\left\{\boldsymbol{\Sigma}_{n}^{-1}-\boldsymbol{\Sigma}_{n}^{-1} \mathbf{s}\left[\mathbf{s}^{H} \boldsymbol{\Sigma}_{n}^{-1} \mathbf{s}+\left(1-v_{n}\right)^{-1}\right]^{-1} \mathbf{s}^{H} \boldsymbol{\Sigma}_{n}^{-1}\right\} \mathbf{s} \\
& =\mathbf{f}_{n}-\mathbf{f}_{n}\left[\mathbf{f}_{n}^{H} \mathbf{s}+\left(1-v_{n}\right)^{-1}\right]^{-1} \mathbf{f}_{n}^{H} \mathbf{s} \\
& =\left[1+\left(1-v_{n}\right) \mathbf{f}_{n}^{H} \mathbf{s}\right]^{-1} \mathbf{f}_{n} \\
& =K_{n} \cdot \mathbf{f}_{n}
\end{aligned}
$$

sendo $K_{n} \triangleq\left[1+\left(1-v_{n}\right) \mathbf{f}_{n}^{H} \mathbf{s}\right]^{-1}$.

Obtém-se assim $\mathbf{f}_{n}^{\prime}$ como o produto de $\mathbf{f}_{n}$ por um escalar. A estimativa $\hat{z}_{n}$ pode então ser expressa por:

$$
\hat{z}_{n}=K_{n} \cdot \mathbf{f}_{n}^{H}\left(\mathbf{y}_{n}-\overline{\mathbf{y}}_{n}+\bar{z}_{n} \mathbf{s}\right)
$$

As estatísticas das LLR ficam sendo:

$$
\begin{gathered}
\mu_{n, i}=K_{n} \cdot \alpha_{i} \cdot \mathbf{f}_{n}^{H} \mathbf{s} \\
\sigma_{n, i}^{2}=K_{n}^{2} \cdot \mathbf{f}_{n}^{H} \mathbf{s}\left(1-v_{n} \mathbf{s}^{H} \mathbf{f}_{n}\right) .
\end{gathered}
$$

E a informação extrínseca de saída:

$$
L_{e}^{E}\left(c_{n, j}\right)=\ln \frac{\sum_{\forall \mathbf{s}_{i}: s_{i, j}=0} \exp \left(-\rho_{n, i}+\sum_{\forall j^{\prime}: j^{\prime} \neq j} \tilde{s}_{i, j^{\prime}}\right)}{\sum_{\forall \mathbf{s}_{i}: s_{i, j}=1} \exp \left(-\rho_{n, i}+\sum_{\forall j^{\prime}: j^{\prime} \neq j} \tilde{s}_{i, j^{\prime}}\right)}
$$

onde

$$
\rho_{n, i} \triangleq \frac{\left|\hat{x}_{n}-\mu_{n, i}\right|^{2}}{\sigma_{n, i}^{2}}=\frac{\left|\mathbf{f}_{n}^{H}\left(\mathbf{y}_{n}-\overline{\mathbf{y}}_{n}+\bar{x}_{n} \mathbf{s}\right)-\alpha_{i} \mathbf{f}_{n}^{H} \mathbf{s}\right|^{2}}{\mathbf{f}_{n}^{H} \mathbf{s}-v_{n} \mathbf{f}_{n}^{H} \mathbf{s s}^{H} \mathbf{f}_{n}}
$$

Na Figura 2 é representado o diagrama em blocos do equalizador MMSE para canais variantes no tempo juntamente com o estimador de canal.

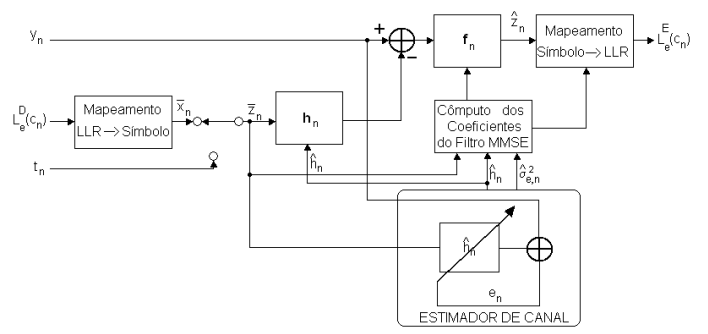

Fig. 2. Estrutura de um equalizador SISO MMSE para canais variantes e conexão com um estimador de canal.

É possível obter um algoritmo recursivo de forma a evitar o cálculo da inversa utilizando um particionamento adequado conforme detalhado em [8].

A inicialização do cálculo recursivo pode ser feita durante um preâmbulo de símbolos conhecidos onde a primeira inversa de $\Sigma_{n}$ a ser calculada torna-se trivial já que $V_{n}=\mathbf{0}$.

\section{Algoritmos Propostos}

A busca por baixa complexidade nos algoritmos de equalizadores SISO pode se concentrar em maneiras de se calcular os coeficientes $f_{n}$ do filtro linear apresentado na Figura 2, visando obter desempenho próximo do obtido com a solução ótima porém com ganhos computacionais.

O cálculo mais oneroso a ser realizado é o cálculo da inversa da matriz $\boldsymbol{\Sigma}_{\mathbf{n}}$, presente em (11). Apesar da forma 
recursiva apresentada em [8], este cálculo pode continuar muito complexo para implementações em tempo real.

O primeiro algoritmo proposto baseia-se no cálculo de $\boldsymbol{\Sigma}_{n}^{-1}$ uma vez apenas a cada quadro de dados. Essa estratégia permite que o processamento do cálculo da inversa seja diluído ao longo do tempo referente a um quadro de dados e um de treinamento, introduzindo apenas um retardo referente a este intervalo de tempo, que poderá não ser significativo, dependendo da relação do tamanho do entrelaçador com o tamanho dos quadros.

Com essa abordagem, o processamento para cálculo da inversa pode ser realizado conjuntamente com outras operações do processador, como, por exemplo, durante o período de treinamento, quando a demanda por processamento é menor, já que não há a necessidade de se calcular as estimativas de símbolos nem as LLR extrínsecas de saída, estando em operação apenas o estimador de canal.

Esse procedimento, porém, ignora as variações dos valores de variância de símbolos transmitidos ao longo de um quadro de dados. Para amenizar esta situação a matriz $\mathbf{V}_{n}$ será substituída por um valor médio de variância de símbolos transmitidos $\overline{\mathbf{v}}$ multiplicado por uma matriz identidade. Este valor será a média empírica sobre todos os valores de informação suave a priori de cada bloco de dados a cada iteração. Esse algoritmo leva aqui o nome de MMSE-SV-TV (SV, de Single Variance e TV, de Time Varying).

Deve-se notar, no entanto, que o estimador de canal continua fornecendo estimativas ao longo do quadro de dados. Assim a equação dos coeficientes do filtro SV-TV fica dada por:

$$
\mathbf{f}_{n}=\Upsilon_{u} \mathbf{s}_{n}
$$

onde

$$
\mathbf{\Upsilon}_{u}=\left(\boldsymbol{\Psi}_{u}+\bar{v} \mathbf{H}_{u} \mathbf{H}_{u}^{H}\right)^{-1}
$$

e $u$ indica o instante de tempo referente ao símbolo de treinamento anterior mais próximo. Apesar de $\Upsilon_{u}$ ser constante ao longo de um quadro de dados, o vetor de coeficientes $\mathbf{f}_{n}$ é diferente a cada intervalo de símbolo devido à atualização de $\mathbf{s}_{n}$.

Caso o quadro de dados seja extenso e o canal varie rapidamente, o desempenho do SV-TV pode não ser satisfatório pela estratégia de manter $\boldsymbol{\Upsilon}_{u}$ constante ao longo do quadro. Uma possibilidade para se tentar contornar este problema é realizar uma aproximação de $\boldsymbol{\Sigma}_{n}^{-1}$ a partir de $\boldsymbol{\Sigma}_{n-1}^{-1}$.

Considera-se o uso de $\bar{v}$ como exposto na seção anterior. Além disso, no lugar de utilizar a matriz $\boldsymbol{\Psi}_{n}$ definida em (8), substitui-se esta matriz por uma matriz identidade multiplicada por uma média empírica de valores da variância do erro $e_{n}$ denotada por $\bar{\sigma}_{e}^{2}$, que será calculada ao longo do preâmbulo de cada bloco transmitido. Desta maneira, tem-se:

$$
\boldsymbol{\Sigma}_{n}=\left(\bar{\sigma}_{e}^{2} \mathbf{I}_{N}+\bar{v} \hat{\mathbf{H}}_{n} \hat{\mathbf{H}}_{n}^{H}\right)
$$

A matriz $\boldsymbol{\Sigma}_{n+1}$ pode ser escrita em função de $\boldsymbol{\Sigma}_{n}$ como

$$
\begin{aligned}
\boldsymbol{\Sigma}_{n+1} & =\left(\bar{\sigma}_{e}^{2} \mathbf{I}_{N}+\bar{v} \hat{\mathbf{H}}_{n} \hat{\mathbf{H}}_{n}^{H}+\bar{v} \Delta\left(\hat{\mathbf{H}}_{n} \hat{\mathbf{H}}_{n}^{H}\right)\right) \\
& =\left(\boldsymbol{\Sigma}_{n}+\bar{v} \Delta\left(\hat{\mathbf{H}}_{n} \hat{\mathbf{H}}_{n}^{H}\right)\right)
\end{aligned}
$$

onde $\Delta\left(\hat{\mathbf{H}}_{n} \hat{\mathbf{H}}_{n}^{H}\right) \triangleq \hat{\mathbf{H}}_{n+1} \hat{\mathbf{H}}_{n+1}^{H}-\hat{\mathbf{H}}_{n} \hat{\mathbf{H}}_{n}^{H}$.
Considerando que a diferença, $\mathbf{h}_{n}-\mathbf{h}_{n-1}$, possui norma pequena, pode-se utilizar uma aproximação de primeira ordem para o cálculo da inversa de $\boldsymbol{\Sigma}_{n+1}$ :

$$
\begin{aligned}
\boldsymbol{\Sigma}_{n+1}^{-1} & =\left(\boldsymbol{\Sigma}_{n}+\bar{v} \Delta\left(\hat{\mathbf{H}} \hat{\mathbf{H}}^{H}\right)\right)^{-1} \\
& \approx \boldsymbol{\Sigma}_{n}^{-1}+\bar{v} \boldsymbol{\Sigma}_{n}^{-1} \Delta\left(\hat{\mathbf{H}} \hat{\mathbf{H}}^{H}\right) \boldsymbol{\Sigma}_{n}^{-1}
\end{aligned}
$$

Fica então definido o algoritmo MMSE-ASV-TV (A, de Aproximation), para o qual o cálculo dos coeficientes é dado por:

$$
\mathbf{f}_{n}=\left(\boldsymbol{\Sigma}_{n-1}^{-1}+\bar{v} \boldsymbol{\Sigma}_{n-1}^{-1} \Delta\left(\hat{\mathbf{H}} \hat{\mathbf{H}}^{H}\right) \boldsymbol{\Sigma}_{n-1}^{-1}\right) \mathbf{s}
$$

Desta maneira, basta realizar um único cálculo de inversa e a partir deste momento realizar a atualização empregando a equação anterior.

\section{Resultados de Simulações}

Todas as simulações foram baseadas no MIL-STD-188110A/B. Este padrão especifica requisitos mínimos de desempenho e interoperabilidade para modems operando na faixa de HF com taxas de comunicação entre 75 e 2400 bps. É previsto uma taxa de símbolos no canal de 2400 bauds, constante para todas as taxas de bit. Para tanto, são adotados parâmetros distintos para cada caso, alterando-se a modulação utilizada e, se for o caso, acrescentando-se redundância de bits codificados.

O codificador utilizado foi do tipo convolucional de taxa $R=1 / 2$ e comprimento de restrição 7 , definido pelos polinômios geradores $g_{1}(x)=x^{6}+x^{4}+x^{3}+x+1 \mathrm{e}$ $g_{2}(x)=x^{6}+x^{5}+x^{4}+x^{3}+1$.

A taxa de bits utilizada foi $2400 \mathrm{bps}$, que corresponde ao emprego de modulação 8PSK. O modulador atua então de forma que cada conjunto $\mathbf{c}_{n} \triangleq\left[c_{n, 1} c_{n, 2} \cdots c_{n, Q}\right]$ de bits codificados $c_{n, j}$ é mapeado em um símbolo $x_{n}$ do alfabeto $S=\left\{\alpha_{1}, \alpha_{2}, \cdots, \alpha_{2}\right\}$, onde $\alpha_{i}$ corresponde à sequiência de bits $\mathbf{s}_{i} \triangleq\left[s_{i, 1} s_{i, 2} \cdots s_{i, Q}\right]$.

A estrutura de treinamento é formada pela transmissão de um preâmbulo de sincronismo que terá a duração de um bloco do entrelaçador. Após esse preâmbulo são alternados quadros de dados com quadros de símbolos conhecidos para retreinamento. A relação entre o tamanho do quadro de dados e o de retreinamento depende da taxa de bits escolhida. Para 1200 bps os quadros de dados e de retreinamento conterão 20 símbolos cada. Já para o caso de 2400 bps o quadro de dados será formado por 32 símbolos e o de retreinamento por 16 símbolos. Esta alternância permanece até o final da mensagem a ser transmitida.

São previstos dois tamanhos de entrelaçador: o curto, com duração de 0,6 s, e o longo, com 4,8s. Como a taxa de símbolos é fixada em 2400 bauds, tais durações correspondem a 1440 e 11520 intervalos de símbolos, respectivamente.

Para o levantamento das curvas de desempenho de taxa de erro, foram transmitidos blocos de tamanho igual ao do entrelaçador, sendo cada bloco tratado como uma transmissão independente. Ou seja, o tamanho da mensagem transmitida foi o tamanho de bloco do entrelaçador. Para cada bloco foi adicionado o preâmbulo de sincronismo e os retreinamentos adequados. Este procedimento foi realizado para pelo menos 

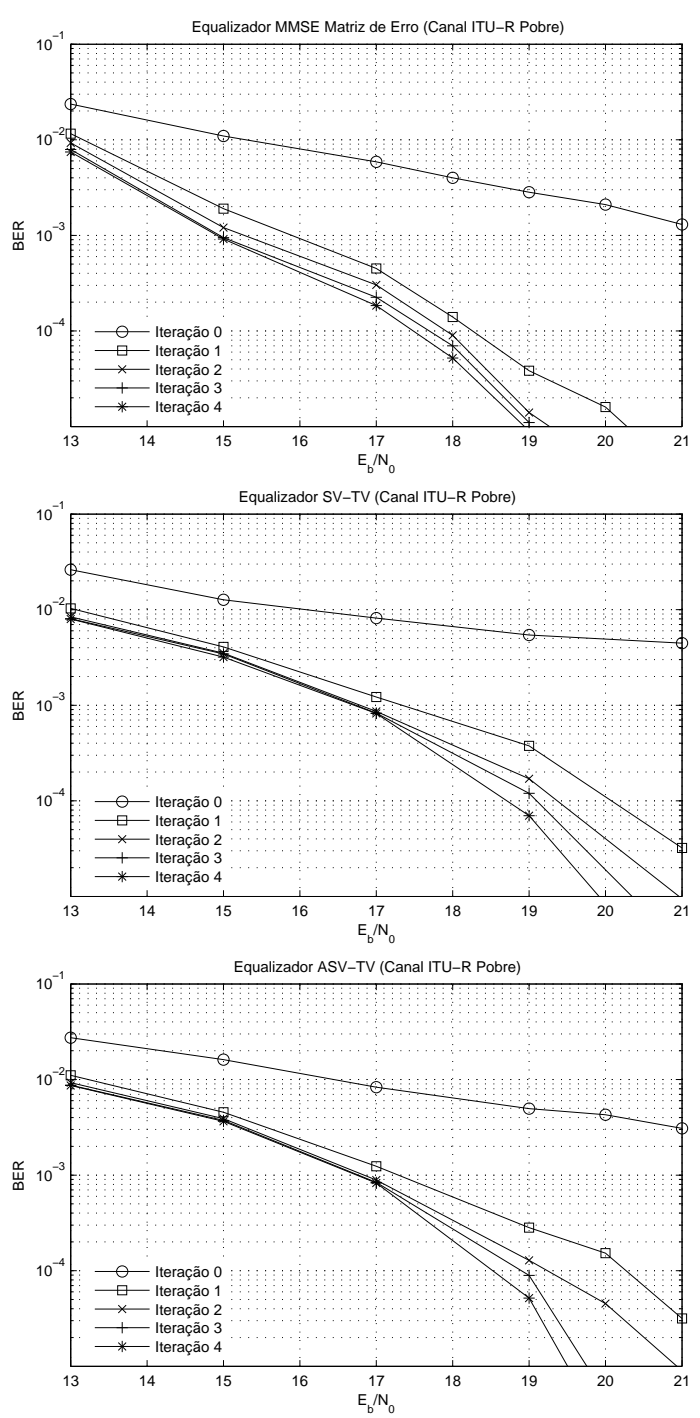

Fig. 3. Taxa de Erro em função da razão $E_{b} / N_{0}$, para canal ITU-R pobre modificado, e comprimento dos quadros de treinamento e dados segundo o MIL-STD-188-110A/B, dos Equalizadores MMSE, SV-TV e ASV-TV.

200 sorteios de canal, garantindo no mínimo 100 erros de transmissão.

O canal utilizado foi uma aproximação do modelo de canal pobre previsto pelo ITU-R, possuindo espectro de desvanecimento gaussiano com um espalhamento Doppler igual a $1 \mathrm{~Hz}$, e formado por dois raios equipotentes independentes. Porém o retardo entre os raios foi utilizado como $2,1 \mathrm{~ms}$, ao invés de $2 \mathrm{~ms}$ como previsto, o que corresponde exatamente a 5 intervalos de símbolos nas condições de simulação.

Para a estimação do canal foi utilizado o algoritmo LMS (Least Mean Squares) com parâmetro de passo igual a 0,02, valor este obtido empiricamente através de levantamento do erro médio quadrático. Para contabilizar o desconhecimento acerca do comprimento da resposta ao impulso do canal foram utilizados 8 coeficientes no estimador.

Para todos os equalizadores implementados foram empregados 24 coeficientes, com $N_{1}=15$ e $N_{2}=8$.

As curvas de desempenho de taxa de erro podem ser vistas na Figura 3.
Os equalizadores SV-TV e ASV-TV apresentam uma degradação da ordem de $1 d B$ com relação ao desempenho do MMSE, tendo o ASV-TV desempenho ligeiramente superior ao SV-TV.

Na Figura 4 estão representadas as curvas de desempenho de taxa de erro para os equalizadores SV-TV e ASV-TV, respectivamente, com o uso do entrelaçador longo, ou seja, 11520 intervalos de símbolo, o que corresponde a 4,8s. Note-se o uso de escalas diferentes quando comparados aos resultados anteriores.

Fica evidenciado nestas figuras o ganho de desempenho obtido pelo aumento de tamanho do entrelaçador, já que tal aumento torna as LLR de entrada mais descorrelacionadas, aproximando-se da situação ideal prevista pelas curvas características.
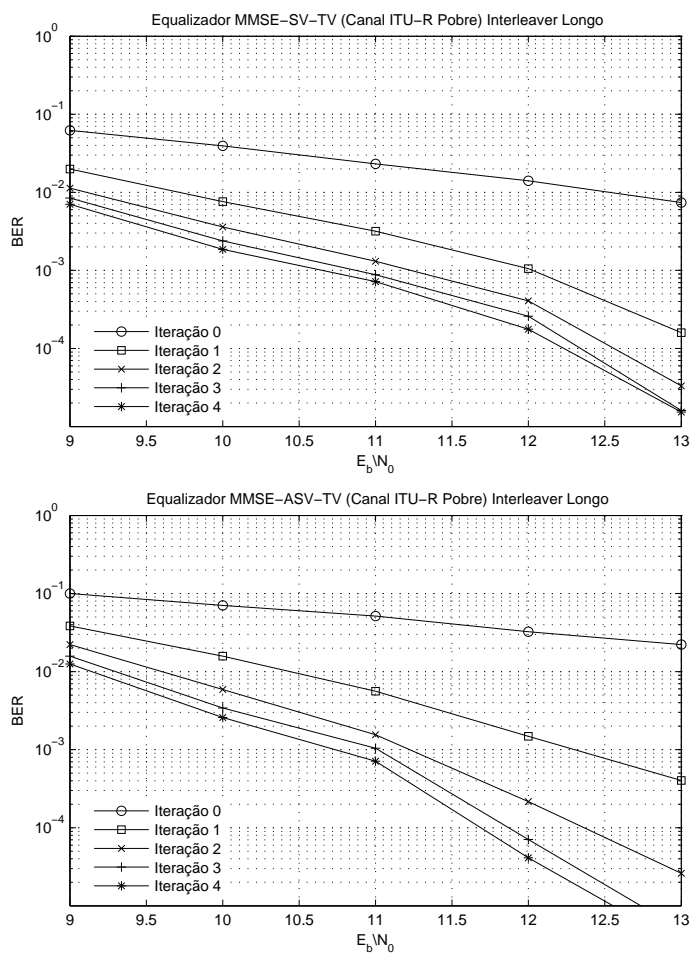

Fig. 4. Taxa de Erro em função da razão $E_{b} / N_{0}$, com entrelaçador longo, para canal ITU-R pobre modificado, e comprimento dos quadros de treinamento e dados segundo o MIL-STD-188-110A/B, dos Equalizadores SV-TV e ASV-TV.

\section{Complexidade Computacional}

Para realizar a análise de complexidade computacional dos algoritmos apresentados foram levadas em consideração somente as operações matemáticas. As demandas por área de memória e o tempo gasto com o acesso às mesmas não foram considerados. Para o cálculo da inversa de uma matriz, foi considerado o algoritmo de Gauss-Jordan.

Os algoritmos apresentados apenas diferem na forma de cálculo dos coeficientes $\mathbf{f}_{n}$, já que o cálculo da variância do erro $e_{n}$ é de responsabilidade do estimador de canal. Sendo assim, para comparar a complexidade dos algoritmos basta observar o número de operações realizadas para o cômputo dos coeficientes do filtro linear a cada instante de tempo. 
Sendo considerado o cálculo recursivo dos coeficientes para o MMSE.

Para o SV-TV, o cálculo da inversa é realizado apenas uma vez a cada conjunto de quadro de dados e de treinamento. Sendo assim, o valor apresentado é o valor médio de operações por símbolo, já que o cálculo pode ser distribuído ao longo do tempo. Os símbolos $K_{T}$ e $K_{D}$, representam respectivamente o tamanho dos blocos de treinamento e de dados. A média para este caso deve ser tomada considerando $K_{T}+K_{D}$, pois, sendo os demais algoritmos recursivos, é necessário o cálculo de $\mathbf{f}_{n}$ mesmo ao longo de quadros de treinamento.

No caso do ASV-TV o cálculo da primeira inversa não é computado para a análise de complexidade, pois é calculado uma única vez.

A Tabela I apresenta o número de operações aritméticas necessário para o cômputo dos coeficientes do filtro linear.

TABELA I

COMPLEXIDADE COMPUTACIONAL DOS EQUALIZADORES SISO PARA O CÁLCULO DE $\mathbf{f}_{n}$.

\begin{tabular}{|c|c|c|}
\hline \multicolumn{2}{|c|}{ Cálculo dos Coeficientes } \\
\hline & $\begin{array}{c}\text { Número } \\
\text { de } \\
\text { Somas }\end{array}$ & $\begin{array}{c}\text { Número } \\
\text { de } \\
\text { Multiplicações }\end{array}$ \\
\hline MMSE & $4 N^{2}-7 N+3$ & $4 N^{2}-2 N-2$ \\
SV-TV & $N^{2}-N+\frac{14 N^{3}-21 N^{2}+6 N^{2} L+7 N}{6\left(K_{T}+K_{D}\right)}$ & $N^{2}+\frac{14 N^{3}+6 N^{2} L-2 N}{6\left(K_{T}+K_{D}\right)}$ \\
\hline ASV-TV & $3 N^{3}+N^{2} L-2 N^{2}-N$ & $3 N^{3}+N^{2} L+N^{2}$ \\
\hline
\end{tabular}

Considerando um processador que consiga efetuar uma divisão no mesmo tempo que uma multiplicação e ignorando as operações de soma, pode-se traçar um gráfico de complexidade para os algoritmos apresentados em função de $N, L, K_{T}$ e $K_{D}$.

Na Figura 5 está representado um gráfico de complexidade considerando-se $K_{T}=16, K_{D}=32$ e $L=8$.

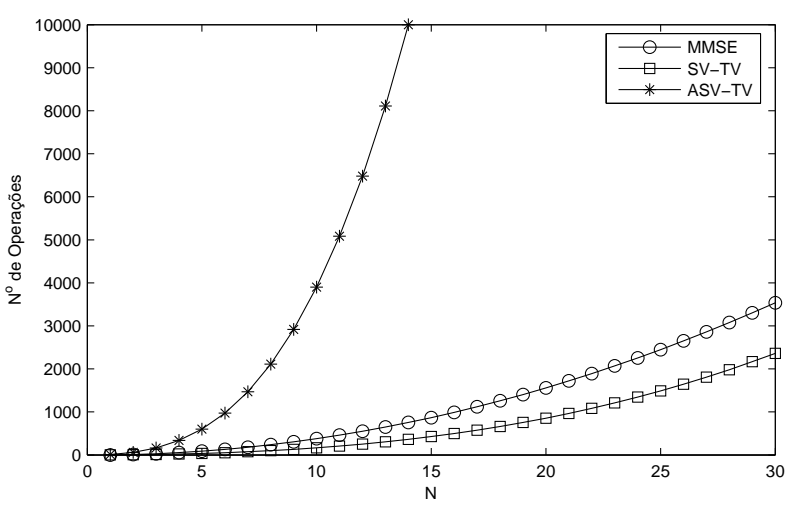

Fig. 5. Comparação do número de operações realizada por cada equalizador para o cálculo de $\mathbf{f}_{n}$.

Conforme pode ser observado na Figura 5 a complexidade do SV-TV fica abaixo da obtida para o caso MMSE, mostrando-se uma boa opção para redução de complexidade. Para o caso do ASV-TV fica claro que a aproximação do cálculo da inversa não supera a obtida pelo uso da recursividade, mostrando não ser uma boa opção a escolha deste último algoritmo.

Porém, apesar de não terem sido explorados neste artigo, alguns algoritmos permitem a redução de complexidade de operações de multiplicação de matrizes, tornando mais competitiva a complexidade do ASV-TV.

Em [10], é citado a existência de um algoritmo que permite a multiplicação de matrizes com complexidade de $O\left(n^{2,38}\right)$, além disso comenta sobre a possibilidade de se realizar as multiplicações com complexidade mais próxima de $O\left(n^{2}\right)$.

Outro ponto passível de ser explorado é a estrutura da matriz de convolução que possui diversos elementos nulos em posições determinadas em função de $N$ e de $L$, o que pode resultar em uma diminuição de complexidade em função destes parâmetros.

\section{CONCLUSÕES}

Baseando-se em algoritmos propostos anteriormente foram obtidos dois algoritmos de equalizadores turbo que utilizam o critério MMSE, permitindo a aplicação a canais de HF.

Foram realizadas avaliações de desempenho utilizandose o padrão MIL-STD-188-110A/B, encontrando-se uma degradação de apenas $1 \mathrm{~dB}$ com relação ao esquema MMSE.

Apesar da pouca perda de desempenho, um dos esquemas propostos apresentou uma redução de complexidade nítida, sendo uma escolha interessante para implementações na faixa de HF.

O impacto do tamanho do entrelaçador também ficou evidenciado pela comparação dos resultados obtidos.

\section{REFERÊNCIAS}

[1] E. Eleftheriou, David D. Falconer, Adaptive Equalization Techniques for HF Channels, in IEEE Journal on Selected Areas in Communications, pp. 238-247, February 1987.

[2] M. Tüchler, R. Otnes, Improved receivers for digital High Frequency waveforms using Turbo equalization, in Proc. Military Communications Conf. (MILCOM), Anaheim, USA, October 2002.

[3] R. Koetter, A. C. Singer, M. Tüchler, Turbo Equalization, invited for Special Issue for the IEEE Signal Processing Magazine, February 2003.

[4] L. Bahl, J. Cocke, F. Jelinek, J. Raviv, Optimal Decoding of Linear Codes for Minimizing Symbol Error Rate, in IEEE Trans. Inform. Theory, vol 20, pp. 284-287, March 1974.

[5] G. Bauch, H. Khorram, and J. Hagenauer, Iterative equalization and decoding in mobile communications systems, in Proc. 2nd European Personal Mobile Commun. Conf. EPMCC'97, pp. 307-312, Bonn., Germany, Sept.-Oct. 1997.

[6] A. Glavieux, C. Laot, and J. Labat, Turbo equalization over a frequency selective channel, in Proc. Int. Symp. On Turbo Codes, pp. 96-102, Brest, France, September 1997.

[7] A. Müller, Simulation of Multipath Fading Channels using the MonteCarlo Method, in IEEE International Conference on Communications, vol. 3, pp. 1536-1540, May 1994.

[8] M. Tüchler, R. Otnes, Low-complexity Turbo equalization for timevarying chanels, in Proc. 55th IEEE Vehicular Technology Conf., Birmingham, AL, USA, May 2002.

[9] S. M. Kay Fundamentals of Statistical Signal Processing: Estimation, Prentice-Hall, Ed. 1, 1993.

[10] Sara Robinson, Toward an Optimal Algorithm for Matrix Multiplication, in SIAM News, vol. 38, nr. 9, November 2005. 